\title{
Amniotic membrane transplantation in a patient with impending perforated corneal ulcer caused by Streptococcus mitis: a case report
}

\section{Fang-Chi Hsiao}

Chang Gung Memorial Hospital Linkou Branch

\section{Yaa-Jyuhn James Meir}

Chang Gung University

\section{Lung-Kun Yeh}

Chang Gung Memorial Hospital Linkou Branch

\section{Hsin-Yuan Tan}

Chang Gung Memorial Hospital Linkou Branch

\section{Ching-Hsi Hsiao}

Chang Gung Memorial Hospital Linkou Branch

\section{Hui-Kang David Ma}

Chang Gung Memorial Hospital Linkou Branch

\section{Wei-Chi Wu}

Chang Gung Memorial Hospital Linkou Branch

Hung-Chi Chen ( $\triangle$ mr3756@cgmh.org.tw)

Chang Gung Memorial Hospital Linkou Branch https://orcid.org/0000-0002-1117-7878

\section{Case report}

Keywords: Persistent corneal ulcer, amniotic membrane transplantation, Streptococcus mitis

Posted Date: March 8th, 2019

DOI: https://doi.org/10.21203/rs.2.434/v1

License: (c) (1) This work is licensed under a Creative Commons Attribution 4.0 International License. Read Full License 


\section{Abstract}

Background: Streptococcus mitis (S. mitis) belongs to the viridan streptococci group which is rarely isolated as a causative pathogen of corneal ulcer. When it causes keratitis, penetrating keratoplasty (PK) might be necessary. Herein, we demonstrated amniotic membrane transplantation (AMT) can be an easier surgery with acceptable outcomes and less complication. Case presentation: A 63-year-old female Taiwanese presented with right persistent corneal ulcer for nine months. Culture from corneal scraping yielded S. mitis. A right eye descemetocele decreased from $3 \mathrm{~mm}$ in diameter to $0.8 \mathrm{~mm}$ after continuous administration of topical Vancomycin and Ceftriaxone for two weeks. Due to retarded healing, AMT was performed. Her corneal erosion healed and became clear gradually. Her visual acuity recovered from counting fingers initially to 20/200 finally 17 months after AMT. Conclusion: This unusual case illustrated that antibiotics plus AMT instead of PK may be an effective alternative treatment to promote epithelialization and reduce inflammation in corneas complicated by S. mitis keratitis.

\section{Background}

Streptococcus mitis (S. mitis) is an alpha-hemolytic, facultative anaerobic species of the viridans group streptococci. It is a commensal of the human oropharynx, and is also found in the skin, gastrointestinal tract, and female genital tract. Though low virulence and pathogenicity are recognized, it is considered as an opportunistic pathogen leading to severe infections including endophthalmitis, infective endocarditis, bacteremia, upper respiratory tract infection and meningitis $[1,2]$.

Moreover, corneal ulcer caused by Streptococcus mitis is rare and has seldom been described. In previous reports, penetrating keratoplasty (PK) was uaually adopt for persistent corneal ulcer [3-5].

As an alternative treatment to reconstruct ocular surface, amniotic membrane transplantation (AMT) has been proposed to promote epithelial healing and reduce neovascularization, inflammation, and scarring, and been demonstrated effective in promoting wound healing and preventing corneal perforation in infectious keratitis [6-9]. In this case, we demonstrated that AMT may be successfully used to treat a patient with an unhealing descemetocele caused by $S$. mitis rather than performing penetrating keratoplasty (PK) which was conventionally adopt before.

\section{Case Presentation}

A 63-year-old Taiwanese Han female, with a history of herpes zoster ophthalmicus 18 years ago, presented to our ophthalmological clinic with a chief complaint of right eye pain. The patient had developed right persistent corneal ulcer for nine months despite use of bi-weekly therapeutic soft contact lenses along with unknown topical agents, which resulted in recurrent symptoms of ocular redness, pain, and blurred vision. Within a few years prior to current event, she reported repeated episodes of right eye redness accompanied with photophobia that dissolved spontaneously about two to three times yearly. Upon initial ocular examination, her visual acuity was counting finger and a $3 \mathrm{~mm} \times 2 \mathrm{~mm}$ central 
epithelial defect with stromal infiltration and a $1 \mathrm{~mm} \times 1 \mathrm{~mm}$ inferonasal paracentral descemetocele of right eye were documented (Figure 1). Famciclovir 250mg, 2 tablets, TID, topical tobramycin ointment $3.5 \mathrm{~g} /$ tube, BID and levofloxacin $0.5 \%, 25 \mathrm{mg} / 5 \mathrm{~mL} /$ bottle, Q1H were prescribed. A subsequent corneal culture yielded $S$. mitis growth. Therefore, hourly topical vancomycin $25 \mathrm{mg} / \mathrm{ml}$ and ceftriaxone $25 \mathrm{mg} / \mathrm{mL}$ were initiated in substitution for previous antiviral and antimicrobials according to the sensitivity test.

The size of descemetocele increased to $3 \mathrm{~mm}$ in diameter initially accompanied with the development of a $1 \mathrm{~mm}$ hypopyon. With continuous administration of topical vancomycin and ceftriaxone for two weeks, the descemetocele gradually shrank to $0.8 \mathrm{~mm} \times 0.8 \mathrm{~mm}$ and the hypopyon resolved (Figure 2).

Superficial manual keratectomy with AMT was performed because of minimal healing and the lack of further shrinkage of descemetocele in spite of intensive topical antibiotics (Figure 3).

During the course of corneal ulcer treatment, the patient reported an abrupt onset of left eye redness with abundant discharge. Pterygium at eight o'clock of cornea and 360 degree chemosis with conjunctival injection (OS) were found. Topical sulfamethoxazole 4\%, TID and fluorometholone $0.1 \%$ QID were used but the symptoms persisted. Therefore, diagnostic aspiration of aqueous (OS) was arranged. Fortunately, no viral DNA or organisms was identified and the severity of chemosis and conjunctival injection had gently improved afterwards.

In post-operative clinic follow-up, the AM remained in situ without further epithelial defects or leakage for up to six months (Figure 4). We switched topical antibiotics to $0.5 \%$ levofloxacin and gradually tapered. The cornea healed and became clear. Visual acuity was 20/200 at the last follow up, when AMT was done 17 months ago.

\section{Discussion And Conclusions}

Well-documented treatments of $S$. mitis keratitis are rare, and most of the reported cases had poor visual outcomes or treated by PK [3-5]. S. mitis is normal flora of the human oropharynx, and is also found in the skin, gastrointestinal tract, and female genital tract. In spite of low virulence and pathogenicity, reports have shown that S. mitis can cause severe infections including endophthalmitis, infective endocarditis, bacteremia, upper respiratory tract infection and meningitis [1, 2]. The organism has been identified in patients with post-surgical endophthalmitis that resulted in poor visual outcomes [10]. In addition, the viridans group streptococci is one of the most common organisms implicated in one rare corneal infectious disease, i.e. infectious crystalline keratopathy [11]. As for infectious keratitis with perforation, Pseudomonas is one of the common isolates [12]. Although corneal ulcer caused by S. mitis has seldom been described, we treated the impending perforated ulcer by antibiotics plus AMT in our case.

Previously in a 10-year review of microbial keratitis from 1972 to 1981, S. mitis was reported in 7\% (3/44) of polymicrobial keratitis and less than $5 \%$ of 133 cases monomicrobial keratitis. Vision of one case was limited to $2 / 200$ by corneal scarring after antibacterial and antifungal therapy. The final vision of another case was 10/200 [3]. In 2005, there was a case report of 39-year-old female presented with S. mitis corneal ulcer with total corneal opacification and a $2.5 \mathrm{~mm} \times 2.5 \mathrm{~mm}$ descemetocele. Antibiotics were 
used, but at last it progressed to perforated cornea and was successfully treated with PK [4]. In 2016, another case of $S$. mitis/oralis corneal ulcer occurred one year after corneal transplantation. Although broad spectrum antibiotics were given, and infection was controlled, the corneal graft was complicated by scar formation. Regrafting was subsequently performed and the new graft finally remained clear [5].

Giving initial topical empiric broad-spectrum antibiotics before available culture data is the general treatment of suppurative keratitis [13]. Surgical treatment options include tissue adhesives, tarsorrhaphy, conjunctival flaps and PK [13]. Management of perforated corneal ulcer or descemetocele involves repair of mechanical disruption, and promotion of reepithelization while reducing inflammation [13, 14]. AMT is one of alternative treatments to reconstruct the ocular surface, and it has been proposed to promote epithelial healing and reduce neovascularization, inflammation, and scarring [6, 7]. Studies have revealed that AMT is effective in promoting wound healing and preventing corneal perforation in infectious keratitis while PK features of removing pathology but has the disadvantage of higher complications $[8,9$, 13].

In this case, we illustrated the clinical and treatment course of an impending perforated corneal ulcer caused by $S$. mitis. We also demonstrated that treatment by antibiotics plus AMT was successful without the need of PK and this could be considered as an alternative treatment of unhealing descemetocele induced by $S$. mitis. Given the current single case report, larger-scale studies are needed for AMT to become one of the standard treatment modalities for persistent corneal ulcer prior to PK.

\section{Abbreviations}

Streptococcus mitis: S. mitis, penetrating keratoplasty: PK, amniotic membrane transplantation: AMT, BID: twice a day, TID: three times a day.

\section{Declarations}

\section{Authors' contributions:}

HCC contributed to the concept and study design.

LKY and HCC treated and enrolled the patient.

FCH and YJM collected the data, made data interpretations.

FCH drafted the manuscript.

All the authors including FCH, YJM, LKY, HYT, CHH, HKM, WCW, and HCC were involved in the critical revision of the manuscript, supervision of the manuscript and final approval of the submission 


\section{Author details:}

${ }^{1}$ Department of Medicine, College of Medicine, Chang Gung University, Taoyuan, Taiwan

${ }^{2}$ Department of Ophthalmology, Chang Gung Memorial Hospital, Linkou, Taiwan

${ }^{3}$ Department of Biomedical Sciences, College of Medicine, Chang Gung University, Taoyuan, Taiwan

${ }^{4}$ Center for Tissue Engineering, Chang Gung Memorial Hospital, Taoyuan, Taiwan

\# The first two authors (Hsiao FC and Meir YJ) contributed equally to this work.

\section{Funding:}

This study was supported by Chang Gung Memorial Hospital, Linkou with award number of CMRPG3G0031-3 and Ministry of Science and Technology, Taiwan with award number of MOST 1072314-B-182A-088-MY3.

\section{Acknowledgements:}

No

\section{Competing interests:}

The authors declare that they have no competing interests.

\section{Availability of data and material:}

All data generated during this case report are included in this published article.

\section{Ethics and consent to participate:}

All procedures performed for the patient were in accordance with the Declaration of Helsinki. Being retrospectively reviewed, this single case report describes the course of the diagnostics and therapy but 
does not include data that can identify the patient, and thus the need for ethical approval was waived.

\section{Consent to publish:}

Written informed consent was obtained from the patient for publication of this case report and any accompanying images.

\section{References}

[1]Mitchell J. Streptococcus mitis: walking the line between commensalism and pathogenesis. Mol Oral Microbiol 2011;26(2):89-98.

[2]Chung JK, Lee SJ. Streptococcus mitis/oralis endophthalmitis management without phakic intraocular lens removal in patient with iris-fixated phakic intraocular lens implantation. BMC Ophthalmol 2014;14(1):92.

[3]Jones DB. Polymicrobial keratitis. Trans Am Ophthalmol Soc 1981;79:153-67.

[4]Nicula C, Szabo I. Complicated corneal ulcer. Case report. Rom J Ophthalmol 2016;60(4):260-3.

[5]Khan ID, Sati A, Arif S, Mehdi I, Bhatt P, Jain V, et al. Streptococcus Mitis/Oralis Corneal Ulcer After Corneal Transplantation. J Basic Clin Med 2016;5(1):8-10.

[6]Hick S, Demers PE, Brunette I, La C, Mabon M, Duchesne B. Amniotic membrane transplantation and fibrin glue in the management of corneal ulcers and perforations: a review of 33 cases. Cornea 2005;24(4):369-77.

[7]Kim JS, Kim JC, Hahn TW, Park WC. Amniotic membrane transplantation in infectious corneal ulcer. Cornea 2001;20(7):720-6.

[8]Chen JH, Ma DH, Tsai RJ. Amniotic membrane transplantation for pseudomonal keratitis with impending perforation. Chang Gung Med J 2002;25(3):144-52.

[9]Chen HC, Tan HY, Hsiao CH, Huang SC, Lin KK, Ma DH. Amniotic membrane transplantation for persistent corneal ulcers and perforations in acute fungal keratitis. Cornea 2006;25(5):564-72.

[10]Durand ML. Endophthalmitis. Clin Microbiol Infect 2013;19(3):227-34.

[11]Khater TT, Jones DB, Wilhelmus KR. Infectious Crystalline Keratopathy Caused by Gram-negative Bacteria. Am J Ophthalmol 1997;124(1):19-23.

[12]Green M, Apel A, Stapleton F. Risk Factors and Causative Organisms in Microbial Keratitis. Cornea 2008;27(1):22-7. 
[13]WHO Regional Office for South-East Asia. Guidelines for the management of corneal ulcer at primary, secondary and tertiary care health facilities in the South-East Asia region. 2004.

[14]Dakhil TAB, Stone DU, Gritz DC. Adjunctive Therapies for Bacterial Keratitis. Middle East Afr J Ophthalmol 2017;24(1):11-7.

\section{Figures}

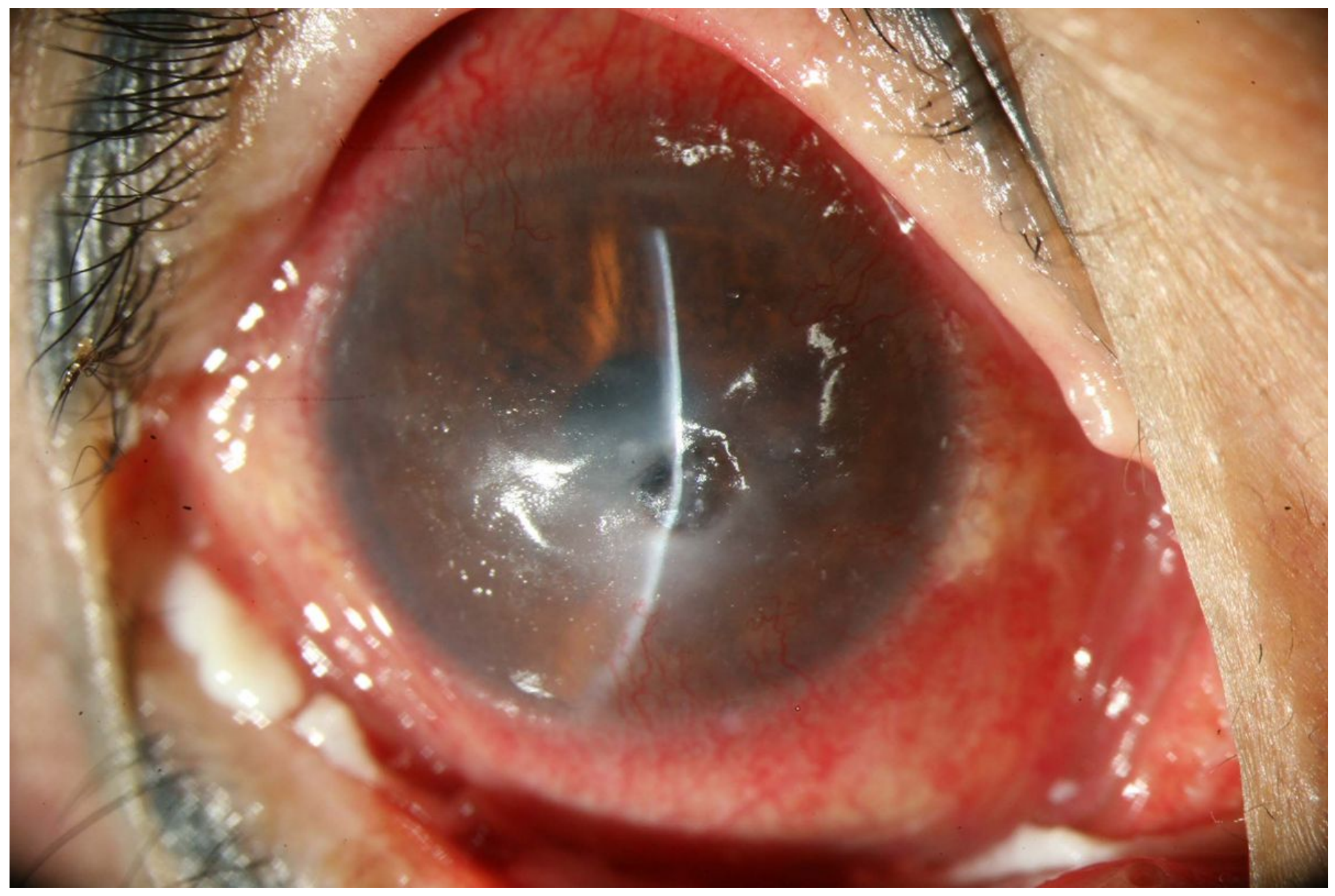

\section{Figure 1}

At initial ocular examination, a $3 \mathrm{~mm} \times 2 \mathrm{~mm}$ central epithelial defect with stromal infiltration and a $1 \mathrm{~mm}$ $\times 1 \mathrm{~mm}$ inferonasal paracentral descemetocele (OD). 


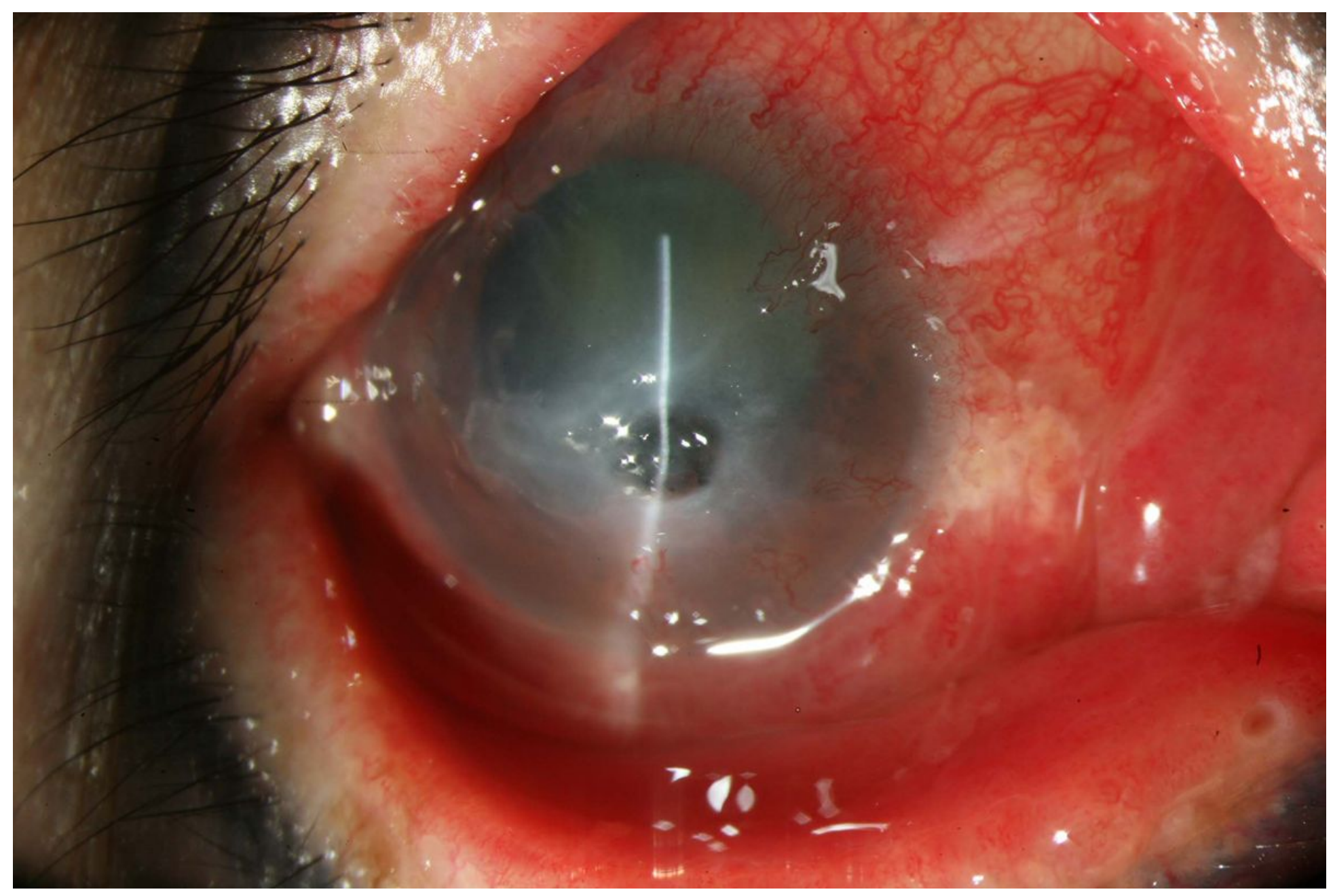

\section{Figure 2}

After continuous administration of topical vancomycin and ceftriaxone for two weeks, descemetocele gradually shrank to $0.8 \mathrm{~mm} \times 0.8 \mathrm{~mm}$ and the hypopyon resolved. 


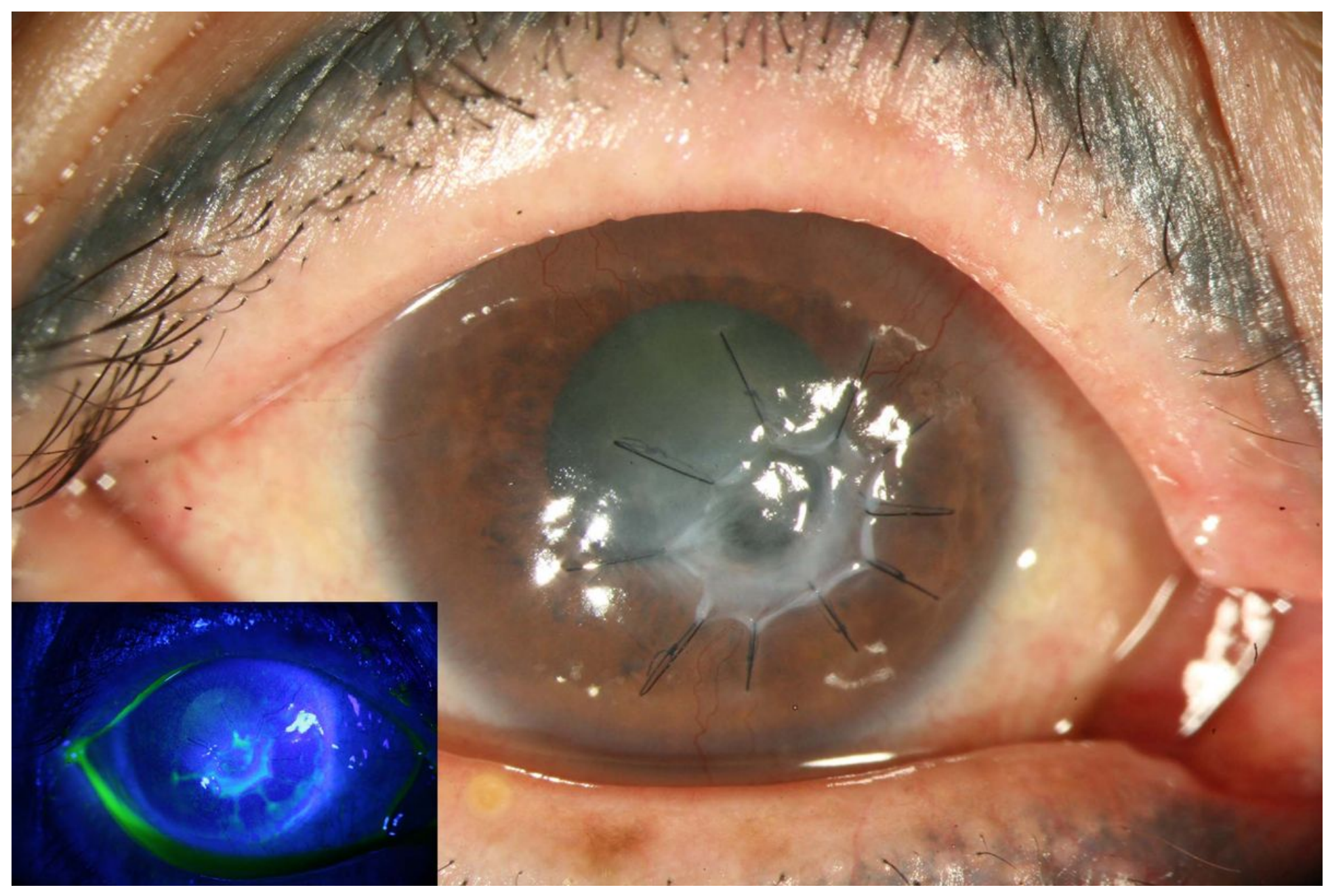

\section{Figure 3}

With superficial manual keratectomy with AMT, the descemetocele was successfully repaired with smooth epithelialization. 


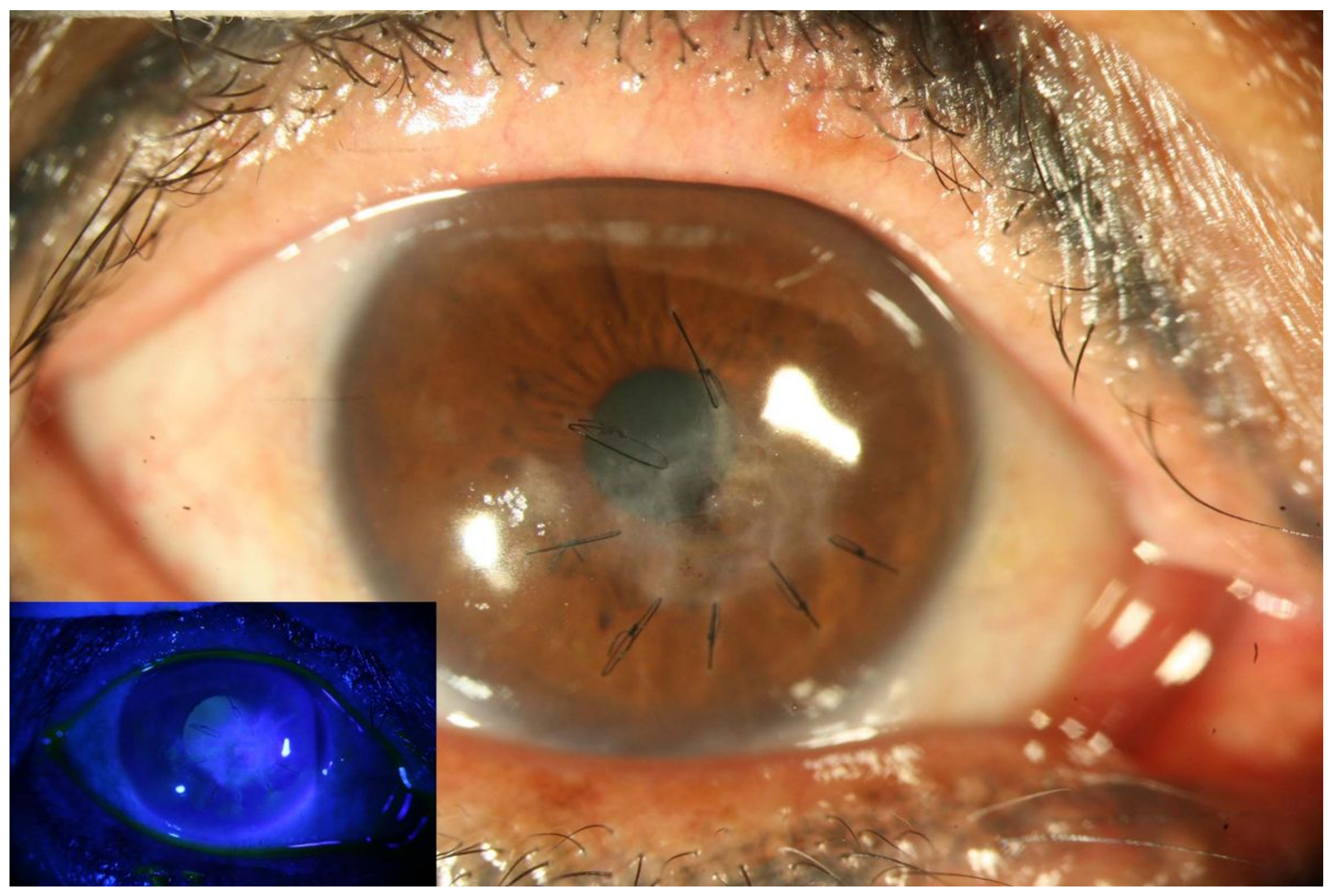

\section{Figure 4}

In post-operative follow-up, the AM remained in situ without further epithelial defects or leakage for up to nine months.

\section{Supplementary Files}

This is a list of supplementary files associated with this preprint. Click to download.

- supplement1.docx 